\title{
Study on Jets Stabilized by Inserting Internal Flow Resistances for the Liquid Metal Divertor in the Helical Fusion Reactor
}

\author{
Takeru OHGO ${ }^{1)}$, Junichi MIYAZAWA ${ }^{1,2)}$, Takuya GOTO ${ }^{1,2)}$ and Takanori MURASE ${ }^{2)}$ \\ 1) The Graduate University for Advanced Studies, 322-6 Oroshi, Toki, Gifu 509-5292, Japan \\ ${ }^{2)}$ National Institute for Fusion Science, 322-6 Oroshi, Toki, Gifu 509-5292, Japan
}

(Received 1 October 2017 / Accepted 18 December 2017)

\begin{abstract}
The stabilization effect of the Internal Flow Resistances (IFR) inserted into water jets has been investigated in order to apply the liquid metal divertor to the helical fusion reactor, FFHR. A high heat load exceeding $20 \mathrm{MW} / \mathrm{m}^{2}$ is one of the important issues for the divertor in the nuclear fusion reactor. A new concept of ergodic limiter/divertor called the REVOLVER-D has been proposed for FFHR. This divertor is expected to tolerate a high heat load larger than a few tens of $\mathrm{MW} / \mathrm{m}^{2}$. Jets narrow after acceleration by gravity and transform to droplets due to the surface tension instability. Stabilization of jets is an important issue for realizing the REVOLVER-D. In this paper, stabilization of jet by various IFR has been examined. Here, the "sheath jet" is defined as a jet with an IFR inside. Experiments on sheath jet have been carried out and the stabilization effect of IFR has been confirmed. Numerical simulations on sheath jet using ANSYS have been carried out. It is shown that the velocity of the sheath jet reaches the terminal velocity, which is proportional to $0.36 \pm 0.01$ power of the flow rate and $-0.26 \pm 0.01$ power of the outer circumference of the IFR.
\end{abstract}

(c) 2018 The Japan Society of Plasma Science and Nuclear Fusion Research

Keywords: divertor, liquid metal, fluid dynamics, jet, finite element method

DOI: $10.1585 / \mathrm{pfr} .13 .1405003$

\section{Introduction}

The divertor in a fusion reactor is a component which removes the heat flux emitted from the plasma and exhausts the outflowing fuel and impurity particles. In the deuterium-tritium reaction, neutrons and helium particles are produced. The helium atoms must be exhausted since these dilute and cool the plasma by enhancing the radiative loss. In the magnetic confinement fusion reactor, the magnetic lines of force finally reach the divertor. Ions move to the divertor along the magnetic lines of force, and finally are neutralized and exhausted at the divertor.

A high heat load over $20 \mathrm{MW} / \mathrm{m}^{2}$ is expected for the divertor in a typical nuclear fusion reactor. This is larger than the tolerable heat load of the "state-of-the-art" divertor being developed for ITER [1]. Conventional solid divertors must realize "divertor detachment" in order to reduce the heat load [2]. However, it is difficult to sustain divertor detachment in steady state. In the fusion reactor, higher neutron and $\gamma$-ray irradiations than in LHD make the performances of measurements and actuators for divertor detachment poor [3]. Instead of the conventional solid divertor, the liquid metal divertor concept is attracting attention. There are many proposed designs utilizing liquid metals as the plasma facing components: the free surface of liquid metals on the first wall [4], Capillary Pore System (CPS) [5-7] and others. The flow velocity of the liquid metal in these examples is as slow as less than $0.1 \mathrm{~m} / \mathrm{s}$. It is possible to increase the tolerable heat load by increasing the flow velocity [8]. The devices utilizing free-falling jets have been also proposed $[9,10]$.

A new concept named the REVOLVER-D (Reactor oriented Effectively VOLumetric VERtical Divertor) consisting of liquid metal shower jets has been proposed for the helical fusion reactor FFHR [3,11]. The REVOLVER$\mathrm{D}$ consists of molten tin shower jets inserted into the ergodic region of the plasma as a limiter/divertor. Tin is selected as the working fluid because of its low vapor pressure, low melting point $\left(\sim 230^{\circ} \mathrm{C}\right)$, low material cost, and high safety (low toxicity, no explosive reaction with water, and high nuclear stability). Neutral particles can be exhausted through the gaps of the shower. The REVOLVER$\mathrm{D}$ is expected to tolerate a high heat load larger than a few tens of $\mathrm{MW} / \mathrm{m}^{2}[12,13]$. Normal jets narrow after acceleration by gravity and readily transform to droplets due to the surface tension instability [14]. Stabilization of jets is one of the important issues for the REVOLVER-D.

In this study, stabilization of the jet by inserting the IFR (Internal Flow Resistance) of, for example, wires, tapes, and chains, has been examined. Here, the "sheath jet" is defined as a jet with an IFR inside. Results of the sheath jet experiment and numerical simulation by ANSYS are described in Secs. 2 and 3, respectively. The summary is given in Sec. 4 . 
Table 1 Comparison of the characteristics between water and liquid metals [15-18].

\begin{tabular}{cccccc}
\hline & $\begin{array}{c}\text { Melting } \\
\text { point }\left({ }^{\circ} \mathrm{C}\right)\end{array}$ & $\begin{array}{c}\text { Kinematic viscosity } \\
\left(\mathrm{m}^{2} \mathrm{~s}^{-1}\right)\end{array}$ & $\begin{array}{c}\text { Density } \\
\left(\mathrm{kg} \mathrm{m}^{-3}\right)\end{array}$ & $\begin{array}{c}\text { Dynamic Viscosity } \\
\left(\mathrm{kg} \mathrm{m}^{-1} \mathrm{~s}^{-1}\right)\end{array}$ & $\begin{array}{c}\text { Surface Tension } \\
\left(\mathrm{kg} \mathrm{s}^{-2}\right)\end{array}$ \\
\hline Water & 0 & $0.89 \times 10^{-6}\left(25^{\circ} \mathrm{C}\right)$ & $997\left(25^{\circ} \mathrm{C}\right)$ & $0.89 \times 10^{-3}\left(25^{\circ} \mathrm{C}\right)$ & $7.20 \times 10^{-2}\left(25^{\circ} \mathrm{C}\right)$ \\
& & $0.24 \times 10^{-6}\left(277^{\circ} \mathrm{C}\right)$ & $6950\left(277^{\circ} \mathrm{C}\right)$ & $1.65 \times 10^{-3}\left(277^{\circ} \mathrm{C}\right)$ & $54.4 \times 10^{-2}\left(232^{\circ} \mathrm{C}\right)$ \\
Tin & 232 & $0.14 \times 10^{-6}\left(627^{\circ} \mathrm{C}\right)$ & $6722\left(627^{\circ} \mathrm{C}\right)$ & $0.95 \times 10^{-3}\left(627^{\circ} \mathrm{C}\right)$ & NA \\
U-alloy78 & 78.8 & $0.24 \times 10^{-6}$ & $8183\left(300^{\circ} \mathrm{C}\right)$ & $2.0 \times 10^{-3}\left(180^{\circ} \mathrm{C}\right)$ & $2.4 \times 10^{-3}\left(20^{\circ} \mathrm{C}\right)$ \\
GaInSn & -19 & $0.37 \times 10^{-6}\left(20^{\circ} \mathrm{C}\right)$ & $6440\left(20^{\circ} \mathrm{C}\right)$ & $53.5 \times 10^{-2}\left(20^{\circ} \mathrm{C}\right)$ \\
\hline
\end{tabular}

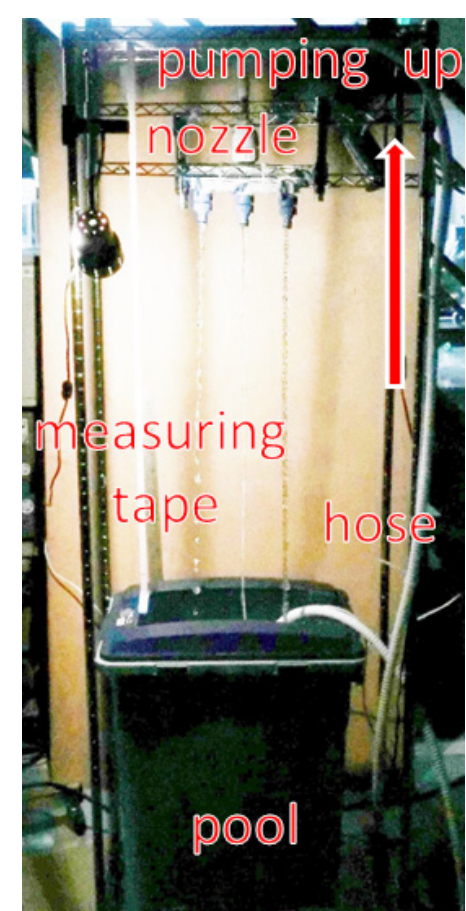

Fig. 1 A water circulation device. This device pumps water from the pool, and issues three jets into the pool. The distance of the nozzle to the pool is $\sim 1 \mathrm{~m}$. The flow rate can be controlled by the regulating valve equipped on each nozzle. The vertical distance from nozzles and the flow width are measured by the measuring tape.

\section{Experiment}

To investigate the stabilization effect of IFR on a jet, controlled jet experiments have been carried out. For this experiment, a water circulation device has been developed as shown in Fig. 1. The aim of this experiment is to investigate the influence of the flow rate, the nozzle width, and the shape of the IFR on the sheath jet characteristics. In this study, water is used in the experiment instead of the molten tin for reasons of safety and experimental cost. The characteristics of water and liquid metals are compared in Table 1. The dynamic viscosity of water is similar to that of tin. On the other hand, the density of water is $\sim 1 / 7$ times smaller. Therefore, the kinematic viscosity of water is 3.7 7 times larger than that of tin. The surface tension of water is $\sim 1 / 7$ times smaller than that of tin. The water

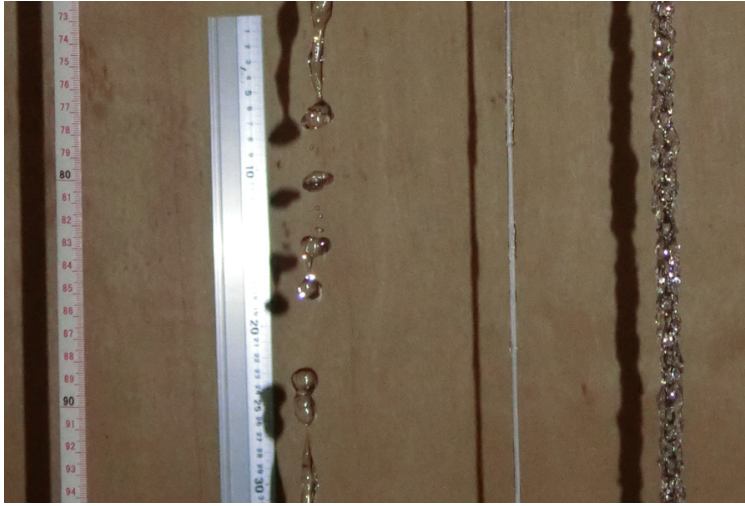

Fig. 2 The photo of a normal jet (left), a sheath jet with a wood pole (center), and a sheath jet with chain (right). The exposure time of this photo was $0.001 \mathrm{~s}$.

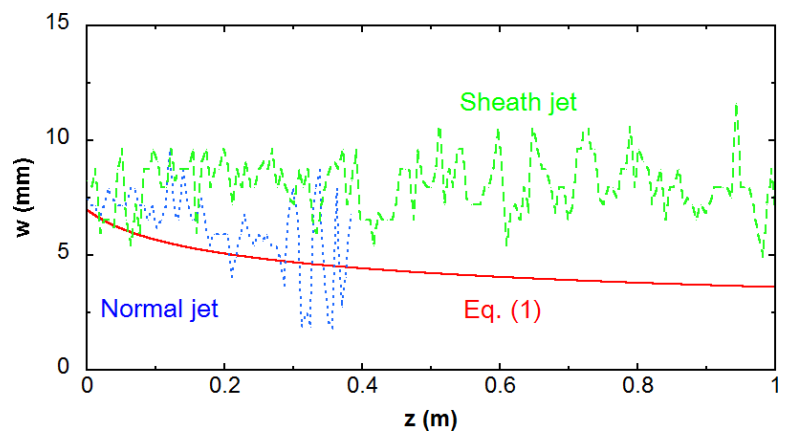

Fig. 3 Comparison of the flow width of the normal jet and the sheath jet as a function of the vertical distance. Dotted line denotes the case of the normal jet, dashed line denotes the case of the sheath jet, and the solid line denotes the theoretical formula for the normal jet (Eq. (1)).

circulation device pumps water from the pool, and issues three jets to the pool. The distance of the nozzle to the pool is $\sim 1 \mathrm{~m}$. The flow rate can be controlled by the regulating valve equipped on each nozzle.

Figure 2 is a close up view of a free-falling jet (left) and sheath jets with a pole (center) or a chain (right) as the IFR. As is clearly seen in Fig. 2, the normal jet easily transforms to droplets, whereas the droplet formation can be avoided by inserting the IFR.

Figure 3 shows the experimental result of the compar- 
ison between the normal jet and the sheath jet. The width and the vertical distance are measured by the tape measure in Fig. 2. This experiment was conducted using a chain as the IFR. The solid line in Fig. 3 denotes the theoretical formula of the flow width in a normal jet, $w$, given by

$$
w=\pi^{-\frac{1}{2}} Q^{\frac{1}{2}}\left(\frac{Q^{2}}{\pi^{2} d^{4}}+2 g z\right)^{-\frac{1}{4}},
$$

where $d, z$, and $g$ are the inner diameter of the nozzle, the vertical distance, and the acceleration of gravity, respectively. The flow rate, $Q$, and the flow velocity, $v$, are given as follows,

$$
\begin{aligned}
& Q=\pi d^{2} v_{0}=\pi w^{2} v, \\
& v=\sqrt{v_{0}^{2}+2 g z}
\end{aligned}
$$

where $v_{0}$ is the initial velocity at the nozzle exit. In the case of the normal jet, the flow width narrowed as predicted by Eq. (1). Finally, a large fluctuation appeared at $z \sim 0.3 \mathrm{~m}$. This fluctuation is related to droplet formation. In the case of the sheath jet, the flow width was roughly constant and no droplet formation was observed. The constant flow width means that the flow velocity of the sheath jet is constant. That is, the flow velocity of a sheath jet becomes the terminal velocity, $v_{\mathrm{t}}$.

In this experiment using a small water circulation device, the flow velocity was limited to less than $\sim 2 \mathrm{~m} / \mathrm{s}$. In the case of the REVOLVER-D, on the other hand, the flow velocity of $\sim 4 \mathrm{~m} / \mathrm{s}$ is needed to assure the heat load tolerance [3]. To bridge this gap, numerical simulation has been conducted.

\section{Numerical Simulation}

To investigate the basic characteristics of the sheath jet, steady-state numerical simulation using the finite element method code, ANSYS, has been carried out. Figure 4 shows the simulated model of the water sheath jet with a pole inside as the IFR. This model corresponds to the experiment described in the previous section. The calculation region is a cylinder of $140 \mathrm{~mm}$ in radius, $10 \mathrm{~m}$ in height. The cylinder is filled with $1 \mathrm{~atm}$ air. A pole with various radii is placed at the center of the cylinder. The boundary conditions at the side wall of the pole were set as the nonslip wall. The nozzle was defined as the circle around the pole at the center of the cylinder top surface. The working fluid was issued from the circle except for the pole region at a uniform initial velocity. The direction of the gravity force is in the $z$ direction. The nozzle width, the IFR width and the initial velocity were varied in the simulation.

Figure 5 shows profiles of the water volume fraction and the velocity obtained by the numerical simulation. Here, in the calculation region, air and water exist. water volume fraction is the ratio of water in each element. The average velocity at each vertical distance of every $100 \mathrm{~mm}$ is obtained by averaging the values of the

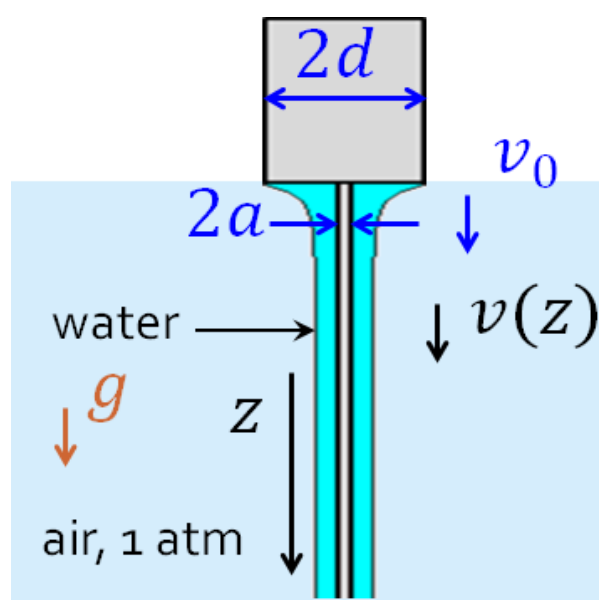

Fig. 4 The simulated model of the water sheath jet with a pole inside as the IFR with a radius of $a$. The calculation region is the cylinder of $140 \mathrm{~mm}$ in radius and $10 \mathrm{~m}$ in height. The nozzle was defined as the circle around the pole at the center of the cylinder top surface with a radius of $d$. The gravity force is working in the $z$ direction. The region without fluid is filled with the air of $1 \mathrm{~atm}$. The nozzle width $d$, the IFR width $a$, and the initial velocity $v_{0}$ are varied in the simulation.

\section{(a)}

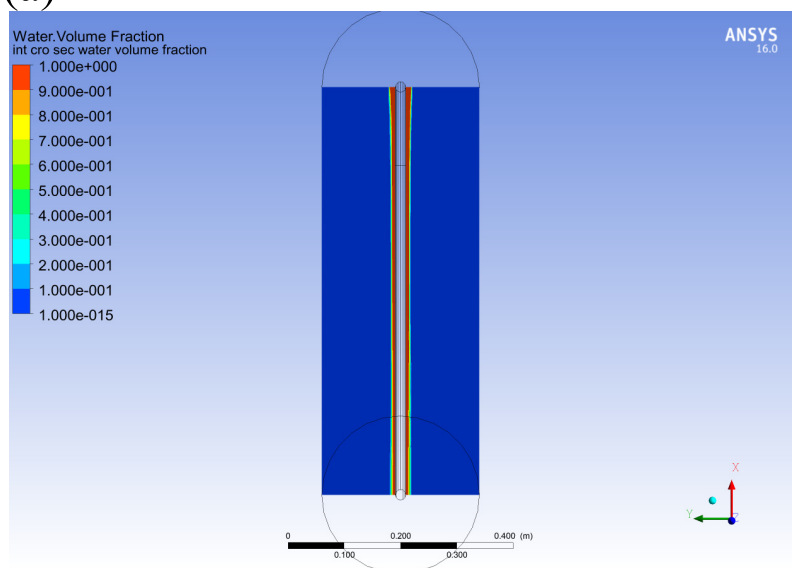

(b)

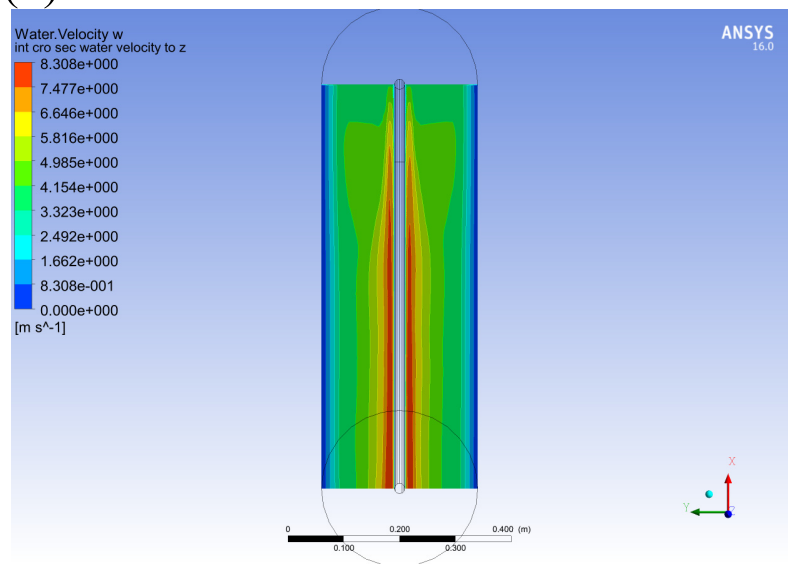

Fig. 5 Profiles of (a) the water volume fraction and (b) the flow velocity in a sheath jet. 


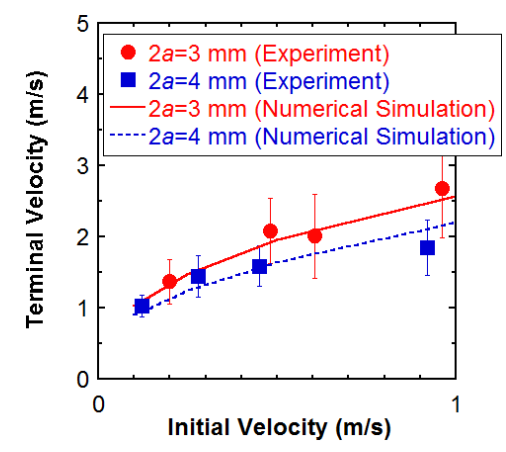

Fig. 6 Comparison of the terminal velocity observed in the experiment (closed symbol) and the numerical simulation (lines), as a function of the initial velocity. Here, $a$ is the IFR radius identical to that in Fig. 4.

(a)

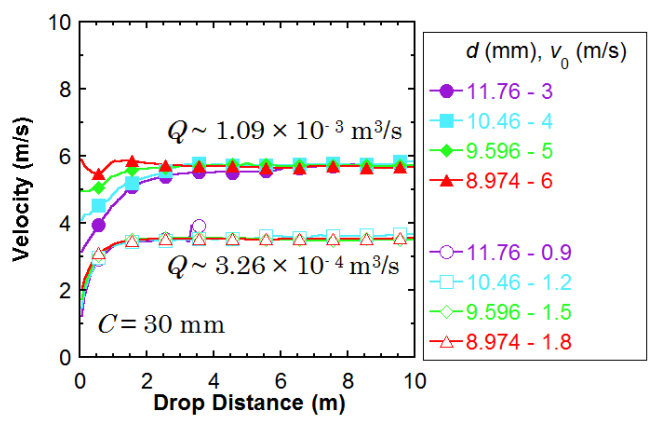

(b)

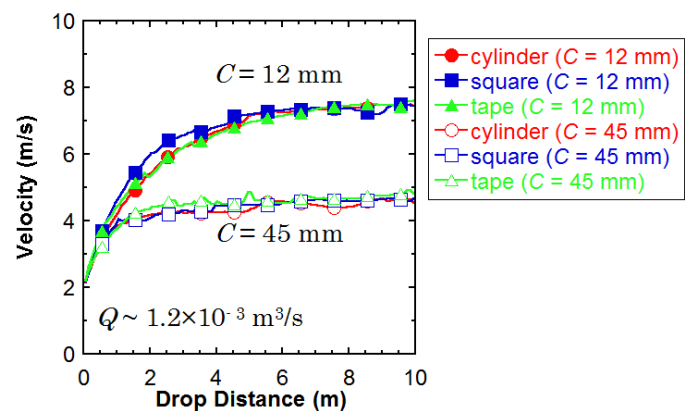

Fig. 7 (a) The simulated velocity with a constant outer circumference of the IFR. The upper lines (closed symbols) have 3.3 times larger flow rates than those of the lower lines (open symbols). Different symbols denote the variation in the nozzle width and initial velocity. (b) The simulated velocity with a constant flow rate. The lower lines (open symbols) have 3.75 times larger outer circumference than those of the upper lines (closed symbols). Different symbols denote the variation in the form of the IFR.

elements of which the water volume fraction is larger than $60 \%$. As will be shown below in Fig. 7, the averaged velocity in the simulation becomes saturated at a large enough vertical distance. This is the terminal velocity as was ob-

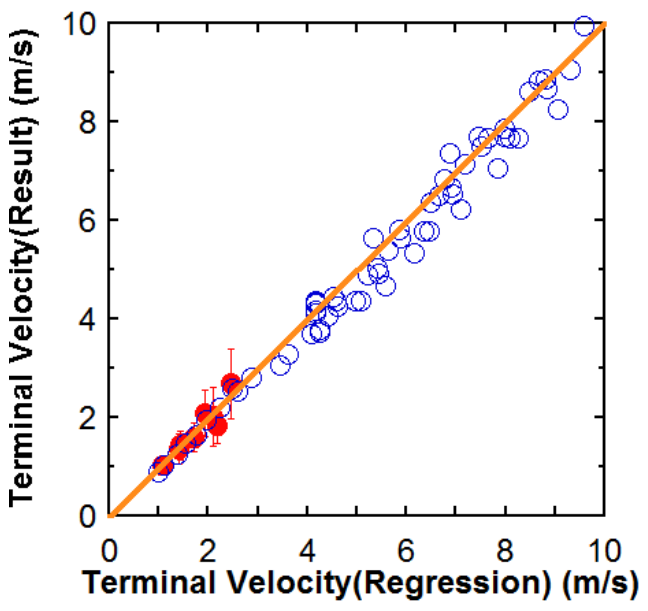

Fig. 8 Comparison between the regression result of Eq. (4) and the results of experiment (closed circles) and numerical simulation (open circles).

served in the experiment, and shown in Fig. 3. Figure 6 compares the relation between $v_{\mathrm{t}}$ and $v_{0}$ in the experiment and the simulation with the IFR radii of $1.5 \mathrm{~mm}$ and $2 \mathrm{~mm}$. In both cases, the nozzle radius was $4.5 \mathrm{~mm}$. It is confirmed that the simulation by ANSYS well agrees with the experiment.

The $v_{\mathrm{t}}$ mainly depends on $Q$ and the outer circumference of the IFR, $C$. Figures 7 (a) and 7 (b) show the dependence of $v_{\mathrm{t}}$ on $Q$ and $C$, respectively. In Fig. 7 (a), the velocity profiles along $z$ of two groups with different $Q$ of $3.26 \times 10^{-4}$ and $1.09 \times 10^{-3} \mathrm{~m}^{3} / \mathrm{s}$ are shown. In each group, the initial velocity and the nozzle width are varied while keeping constant $Q$. An identical $C$ of $30 \mathrm{~mm}$ was used for all cases. The group with the larger $Q$ settles in the larger $v_{\mathrm{t}}$. In Fig. 7 (b), the velocity profiles along $z$ of two groups with different $C$ of 12 and $45 \mathrm{~mm}$ are shown. In each group, the shape of the IFR is varied while maintaining constant $C$. An identical $Q$ of $1.2 \times 10^{-3} \mathrm{~m}^{3} / \mathrm{s}$ was used for all cases. The group with the smaller $C$ settles in the larger $v_{\mathrm{t}}$.

According to these results, the regression analysis on $v_{\mathrm{t}}$ using $Q$ and $C$ has been carried out. The result is

$$
v_{\mathrm{t}}=(2.4 \pm 0.1) \times 10 C^{-0.26 \pm 0.01} Q^{0.36 \pm 0.01} .
$$

Equation (4) and the results of the experiment and simulation are compared in Fig. 8. Both results are well reproduced by Eq. (4).

\section{Summary}

The stabilization effect of the IFR on the jet has been examined and the basic properties of the sheath jet have been investigated. It was found that jets can be stabilized by inserting an IFR. It has been shown that the velocity of the sheath jet saturates at $v_{\mathrm{t}}$. From the simulation results, the scaling of $v_{\mathrm{t}}$ has been obtained as $v_{\mathrm{t}} \sim$ $C^{-0.26 \pm 0.01} Q^{0.36 \pm 0.01}$. This scaling also well reproduces the 
experimental result using water. For REVOLVER-D, liquid tin will be used as a working fluid. Water is similar to tin on the dynamic viscosity. On the other hand, the density of tin is 7 times higher than that of water, and the surface tension also differs. The difference between the water and tin sheath jets should be investigated in the future study.

\section{Acknowledgments}

This work was supported by NIFS budget number NIFS15UFFF038.

[1] J. Schlosser et al., Nucl. Fusion 45, 512 (2005).

[2] J. Miyazawa et al., Fusion Sci. Technol. 58, 200 (2010).

[3] J. Miyazawa et al., Conceptual design of a liquid metal limiter/divertor system for the FFHR-d1, Fusion Eng. Des. (2017), http://dx.doi.org/10.1016/j.fusengdes.2017.07.003

[4] R.W. Moir, Part. Accel. 37, 467 (1992).

[5] S. Mirnov, J. Nucl. Mater. 390, 876 (2009).
[6] G. Mazzitelli, M.L. Apicella, A. Alexeyev and FTU Team, Fusion Eng. Des. 86, 580 (2011).

[7] F.L. Tabarés et al., J. Nucl. Mater. 463, 1142 (2015).

[8] M. Ida et al., Fusion Eng. Des. 63, 333 (2002).

[9] S.V. Mirnov, J. Nucl. Mater. 196, 1014 (1992).

[10] F. Okino, R. Kasada and S. Konishi, Fusion Eng. Des. 89, 1054 (2014).

[11] A. Sagara et al., Nucl. Fusion 57, 086046 (2017).

[12] S. Masuzaki et al., Nucl. Fusion 42, 750 (2002).

[13] M. Shoji et al., Fusion Sci. Technol. 58, 208 (2010).

[14] F. Okino, K. Noborio, Y. Tamamoto and S. Konishi, Fusion Eng. Des. 87, 1014 (2012).

[15] M.J. Assael et al., J. Phys. Chem. Ref. Data 39, No. 3, 033105 (2010).

[16] Galinstan - Wikipedia, https://en.wikipedia.org/wiki/ Galinstan

[17] J.C. Crittenden et al., MWH's Water Treatment: Principles and Design, Third Edition (John Wiley \& Sons, Inc, 2012)

[18] E. Matsuo, The PhD Dissertation of University of Tsukuba, 2013. http://hdl.handle.net/2241/00122776 